\title{
Finite Bias Calculations to Model Interface Dipoles in Electrochemical Cells at the Atomic Scale
}

Martin Hangaard Hansen $^{\mathrm{a}, \mathrm{b}}$, Chengjun Jin ${ }^{\mathrm{a}}$, Kristian S. Thygesen ${ }^{\mathrm{a}}$, Jan Rossmeisl ${ }^{\mathrm{b}}$

${ }^{a}$ Technical University of Denmark, Fysikvej Building 307, DK-2800 Kgs. Lyngby

${ }^{b}$ Department of Chemistry, University of Copenhagen, Universitetsparken 5, DK-2100

København Ø.

\section{Supplementary information}

Atomic structures for the neutral water/slab interfaces were handled through the Atomic Simulation Environment ${ }^{1}$. Calculations of the water/slab interface structures were performed by DFT with the localized atomic orbitals ${ }^{2}$ basis sets implemented in the $\mathrm{GPAW}^{3,4}$ software. The real space grid-point spacing was $0.2 \AA$. All the half cell structures had four layers of water and four FCC(111) layers of gold. In the in-plane directions the $\mathrm{Au}(111)$ surface had a 3 by 4 super cell. 4 by $4 \mathrm{k}$-point sampling ${ }^{5}$ and periodic boundary conditions was used. They were centered in the out-of-plane direction with $10 \AA$ of vacuum on either side and a dipole correction ${ }^{6}$ was applied, in order to keep the electrostatic potentials flat in a vacuum region and measure the work function. A Fermi smearing of $0.1 \mathrm{eV}$ was used and energies were extrapolated to $0 \mathrm{~K}$ in electronic temperature. Exchange and correlation contributions were calculated by the $\mathrm{PBE}^{7}$ functional. The set of various half-cell structures was produced using ab initio molecular dynamics (MD), which varied the water structures, while the top layer was constrained to keep the water molecules confined near the surface. The MD time step was $1 \mathrm{fs}$, and the temperature was kept near $300 \mathrm{~K}$ by a Berendsen thermostat with a time constant of $500 \mathrm{fs}$. A sampling run of 3 ps was sufficient to obtain structures with the variety of work functions that were used here. 
Finite bias calculations were performed for the full cell systems with the non-equilibrium Green's function formalism combined with density functional theory (NEGF+DFT) using the Transiesta code ${ }^{8,9}$. The default double-zeta polarized basis functions and the $\mathrm{PBE}^{6}$ exchangecorrelation functional were employed. A 4 times 4 k-point mesh was sampled for the transverse Brillouin zone in the transport calculations. For the bulk electrodes, a 4 times 4 times 100 k-point mesh was sampled for the Brillouin zone. In all cases, the mesh cutoff was $100 \mathrm{Ry}$.

\section{References}

(1) Bahn, S. R.; Jacobsen, K. W. An Object-Oriented Scripting Interface to a Legacy Electronic Structure Code. Comput. Sci. Eng. 2002, 4, 56-66.

(2) Larsen, A. H.; Vanin, M.; Mortensen, J. J.; Thygesen, K. S.; Jacobsen, K. W. Localized Atomic Basis Set in the Projector Augmented Wave Method. Phys. Rev. B 2009, 80, 195112.

(3) Mortensen, J. J.; Hansen, L. B.; Jacobsen, K. W. Real-Space Grid Implementation of the Projector Augmented Wave Method. Phys. Rev. B 2005, 71, 035109.

(4) Enkovaara, J.; Rostgaard, C.; Mortensen, J. J.; Chen, J.; Dułak, M.; Ferrighi, L.; Gavnholt, J.; Glinsvad, C.; Haikola, V.; Hansen, H. A. Electronic Structure Calculations with GPAW: A Real-Space Implementation of the Projector Augmented-Wave Method. J. Phys.: Condens. Matter 2010, 22, 253202.

(5) Monkhorst, H. J.; Pack, J. D. Special Points for Brillouin-Zone Integrations. Phys. Rev. B 1976, 13, 5188-5192.

(6) Bengtsson, L. Dipole Correction for Surface Supercell Calculations. Phys. Rev. B 1999, 59, 12301.

(7) Perdew, J. P.; Burke, K.; Ernzerhof, M. Generalized Gradient Approximation Made Simple. Phys. Rev. Lett. 1996, 77, 3865-3868.

(8) Brandbyge, M.; Mozos, J.-L.; Ordejón, P.; Taylor, J.; Stokbro, K. Density-Functional Method for Nonequilibrium Electron Transport. Phys. Rev. B 2002, 65, 165401.

(9) Ordejón, P.; Artacho, E.; Soler, J. M. Self-Consistent Order-N Density-Functional Calculations for Very Large Systems. Phys. Rev. B 1996, 53, R10441-R10444. 